\title{
Diagnosis of Faults due to Misfiring of Switches of a Cascaded H-Bridge Multi-level Inverter using Artificial Neural Networks
}

\author{
S. K. Salankayana \\ Dept. of EEE,SRM University \\ Chennai, India
}

\author{
N. Chellammal \\ Dept. of EEE, SRM University \\ Chennai, India
}

\author{
Ravitheja Gurram \\ Dept. of EEE, SRM University \\ Chennai, India
}

\begin{abstract}
This paper presents an artificial neural network based fault identification system for a five-level cascaded H-Bridge multilevel inverter (MLI). A Radial Basis Function (RBF) neural network is trained using radial basis functiontraining algorithm to identify the location of the switch that is misfired at an instant prior to its actual firing time.The proposed fault diagnostic system identifies the fault with a greater accuracy and the results to various input patterns are presented in a tabular format for easy comprehension.
\end{abstract}

Keywords-Artificial Neural Networks, Cascaded H-Bridge Inverter, Multi-Level Inverter,RBF, Radial Basis Function, Switch Misfiring

\section{INTRODUCTION}

Applications in present time require converters with high power and medium voltage ratings. Designing single apparatus for such applications proved to be an expensive and herculean affair as it is troublesome to connect only one semiconductor switch directly across the medium voltage high power grid. As a result, multi-level converter structure has been introduced as an alternative to the conventional converters in medium voltage high power grids. A multi-level converter not only achieves higher power ratings but also, provides a convenient connectivity to the Renewable Energy sources such as the Solar Photovoltaic modules, Wind turbine-generator sets, Fuel cells, etc. [1], [2].

This paper primarily focus on diagnosing of faults on a 5-level cascaded multi-level inverter using a multi-layer radial basis function network trained to identify faults based on the output waveform patterns of the MLI trained using the Radial basis function algorithm.

\section{MULTI-LEVEL INVERTERS}

The origin of the term "Multi-level Inverter" can be traced back as long as the designing of the single-phase four-switch inverter with three voltage levels introducing the third voltage level of $0 \mathrm{~V}$ in the conventional inverter. Hence, a multi-level inverter can be defined as "A Power Semiconductor module that converts a fixed DC input to an AC voltage of variable frequency with three or more voltage levels". The basic concept of a multi-level inverter to achieve higher power rating is to use several series connected switching modules of lower ratings connected to individual DC sources to synthesize staircase AC waveforms with the required number of levels.

Multi-level inverters have the following advantages over the conventional PWM inverters [1]: a Near sinusoidal stepped wave output which results in reduction of $\mathrm{dv} / \mathrm{dt}$ stresses on the switches in turn reducing or eliminating the harmonics and other EMC problems.

× Lower Common-mode voltages

a Distortions in the current drawn by the MLI from the source are reduced resulting in enhanced performance and increased life of the DC sources or other storage components.

\ Wide bandwidth of operation

Multi-level inverters are realized using various topologies such as Cascaded H-bridge Inverter, Diode Clamped MLI, Flying Capacitor MLI, etc. Of the available MLI topologies, Cascaded H-Bridge MLI is most commonly used due to its flexibility in increasing and decreasing of the number of output levels required, less number of components to realize a certain level MLI as compared to the diode clamped or flying capacitor MLIs and the provision of using separate DC sources for each module, enhances the stability and performance of the system [3], [4].Figure. 1 depicts an m-level Cascaded MLI with [ $(m-1) / 2-$ 1] modules of conventional four switch inverters with individual DC sources.

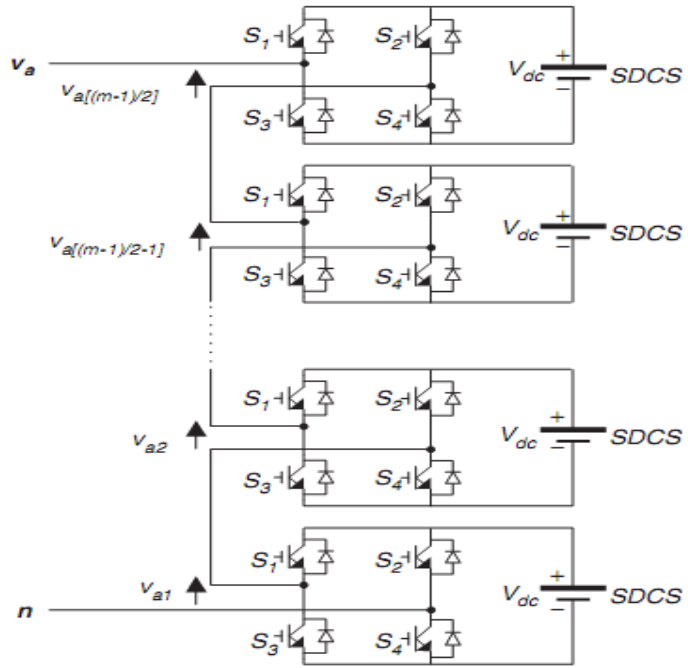

Figure 1.m-level Cascaded H-Bridge MLI

Faults on any system are unavoidable and lead to instability of the system. Hence, the system should be built Fault-Tolerant and should have the ability to recognize the type of the fault that has occurred and take corresponding measures to minimize the effect of fault to the extent maximum possible and help the system to 
stay in its stable state of operation irrespective of the external parasitic factors [5].

A Cascaded H-Bridge MLI is prone to the following faults:

\ Misfire of Switches

× Failure to trigger

a Commutation failure

\ DC Source Short-circuit

× Short-Circuit of Load terminals

\ Overloading

\ Sudden Removal of Load

\ Failure of DC Sources

\section{ARTIFICIAL NEURAL NETWORKS}

With the conventional controllers becoming a cliché due to their slow response time, low precision, higher margin of errors, etc., complex yet simple controllers such as the Artificial Intelligent Controllers, Fuzzy logic controllers, Embedded controllers, control systems on Application Specific ICs, etc. have created their mark in the world of controlling technology. Of all the nonconventional controllers, AI controllers proved to have the maximum accuracy and precision with the lowest error margins (even zero error margins) if trained for the application properly.

Since the introduction of the concept of Artificial Neural Networks in 1943 by Warren McCulloch and Walter Pitts, many architectures and learning laws have been developed and tested for various applications ranging from simple applications such as realization of Boolean logic function to complex applications such as pattern recognition, classification, data interpretation and storage applications, control of various industrial processes etc. [6].

ANN Controllers are designed based on the fascinating architecture of the Human Brain. The human brain is the collection of millions of small basic units called neurons. These neurons collect the input signals, process them for various functions and transmit them to the output units such as muscles. The neurons also act as data storage elements to form the memory unit. Inspired from this concept, the artificial neural networks are designed with artificial neurons as the base units. According to McCulloch and Pitt's model, the artificial neuron is an interconnection of the input channels to a summing junction, where their weighted sum is computed and passed on to the threshold unit which activates the next set of neurons or the output devices based on the threshold response of the computed weighted sum.

Artificial Neural Networks provide efficient response to any kind of input pattern [7]. They can adapt themselves to the input patterns by continuously training themselves by adjusting the weights between the neurons or the layer of neurons which also results in wider operating range. The complex interconnection of the neurons make the ANN fault tolerant as a set of neurons can take over the load of the faulted set of neurons without any considerable change in the performance of the whole system.

\section{FAULTS ON A MULTI-LEVEL INVERTER}

\subsection{Switch Misfiring}

Misfiring of switches is a very common type of fault that occurs on any Power Electronic devices, be it converters or inverters or choppers or complex devices such as the FACTS converters. A switch is said to be misfired when it is triggered at an instant earlier to when it is actually meant to be triggered. Misfiring of a switch in the same leg might lead to a direct short circuit of the source leading to disastrous effects or simply cause the output voltage to drop to zero thus affecting the performance of the load connected and the overall stability of the system.

Hence, misfiring of switches has to be detected as early as possible and compensated for to avoid any instability and degradation of the system.

\subsection{Diagnosis of Faults due Misfiring of Switches}

Detecting a fault that occurred due to misfiring of switches or due to any other fault related to the switching devices in the system can be done using quite a few methods viz., a) Analyzing the harmonic content in the output voltages of the MLI [7], b) Analyzing the output waveform patterns, c) Considering the number of voltage levels per cycle of output [8] c) monitoring the voltages and currents at the switches, d) monitoring the pulses given to the switches and the gate driver circuits, etc. Of these methods, analyzing the output waveforms would yield accurate results quicker than the other methods as each type of fault on any of the switch(s) would leave a unique distortion pattern in the input, which can be directly used for fault diagnosis and compensation.

Using conventional controllers or monitoring equipment, detecting these switch level faults becomes a herculean task and the results may lack accuracy or precision or the computational time to detect these faults is high. In order to overcome these issues, non-conventional controllers such as the ANN controllers, Fuzzy logic controller, etc., are used. This paper deals with the Artificial Neural Network based fault diagnostic system.

\section{RADIAL BASIS FUNCTION NEURAL NETWORKS: ARCHITECTURE \&LEARNING}

\subsection{RBF Neural Network Architecture}

As the concept of ANN evolved, various architectures of the artificial neural networks were developed that expertise in a given kind of applications [9], [10].For instance, the perceptron network works flawlessly with the data that is linearly separable. Whereas, the ADALINE and MADALINE networks work good with hard problems, the BAM, Elman Architecture work better as memory units or data storage networks. The Feed Forward, Cascade Forward architectures [11], [12] and the RBF network can be employed for applications where the network has to deal with continuously varying inputs that take continuous values ranging over the Real domain.

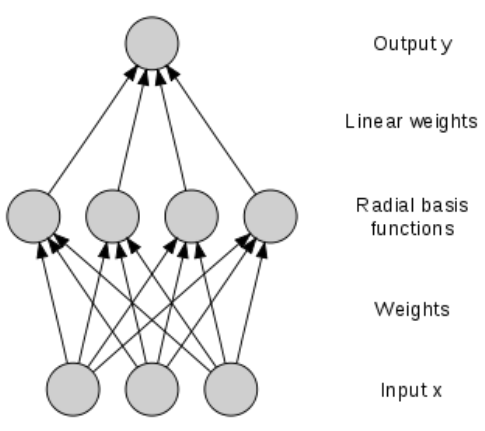

Figure 2. RBF Network architecture 
In this paper, RBF network architecture is implemented. The RBF network as shown in Figure2has three layers, one input layer, a hidden layer and an output layer. The neurons in the input layer are linked to those in the hidden layer through unit weights. The number of neurons in the input layer equals to the number of samples taken in the input data. The hidden layer neurons consist of non-linear radial basis function type activation as shown in Figure 3, which computes its output value based on the Euclidean distance of the weighted sum of the samples from the center vector of the neuron. The number of neurons in the hidden layer is a variable parameter. The neurons are added and removed from the hidden layer during the training process and the optimum number of neurons is fixed along with their centers and the spread factor of the activation function.

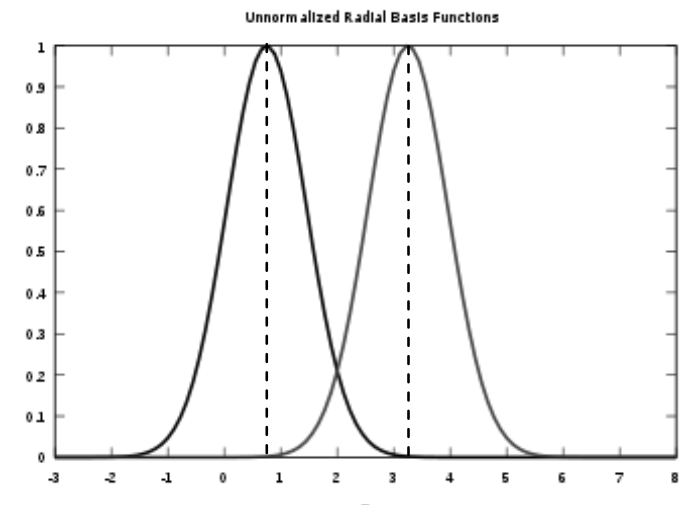

Figure 3. RBF activation functions with centers at $c_{1}=0.75$ and $c_{2}=3.25$

\subsection{Training of a Radial Basis Function Network}

The training of an RBFN involves updating of three main parameters apart from the weights as done in other networks, the parameters being the center vectors of the activation functions, the spread or width parameter of the activation functions and the number of neurons in the hidden layer [13]. The optimal number of hidden layer neurons can be obtained from optimization algorithms such as the particle swarm optimization, evolutionary programming, genetic algorithm, modified genetic algorithm, etc. or they can also be determined by adding a fixed number of neurons (preferably one or two neuron) per epoch, updating the weights and validating the patterns. The number of neurons that give the best fit are considered to be the optimal number of neurons in the hidden layer.

The training algorithm for the RBFN [14]-[15] as stated involves:

Step 1: Computing the number of hidden neurons $\mathbf{N}$ by various optimization techniques mentioned.

Step 2: Compute the cluster centers $\mathbf{c}_{\mathbf{i}}$ using either the K-means clustering technique or using the random subsets of the training points.

Step 3: Interpolate the weights using the relation

$$
[\mathbf{w}]=[\mathbf{G}]^{-1}[\mathbf{x}]
$$

where,

$$
[\mathbf{G}]=[\mathbf{g i j}]=\rho\left(\left\|x_{j}-c_{i}\right\|\right)
$$

and $\rho\left(\left\|x_{j}-c_{i}\right\|\right)$ is the Gaussian function given by

$$
\rho\left(\left\|x_{j}-c_{i}\right\|\right)=\exp \left[-\beta\left\|x_{j}-c_{i}\right\|^{2}\right]
$$

where $\beta$ is the spread parameter.
Step 4: Compute the output of the network given by

$$
\varphi(x)=\sum_{i=1}^{N} w_{i} \rho\left(\left\|x_{j}-c_{i}\right\|\right)
$$

Step 5: Repeat steps 1 to 4 until the best fit between the output $\varphi(x)$ and the targets is reached.

\section{TEST CIRCUITS AND SIMULATONS}

Figure 4 shows the general block diagram of a fault diagnostic system. The output voltages are measured and given as inputs to the ANN controller which identifies the type of fault and provides the gating pulses accordingly to compensate for the fault.

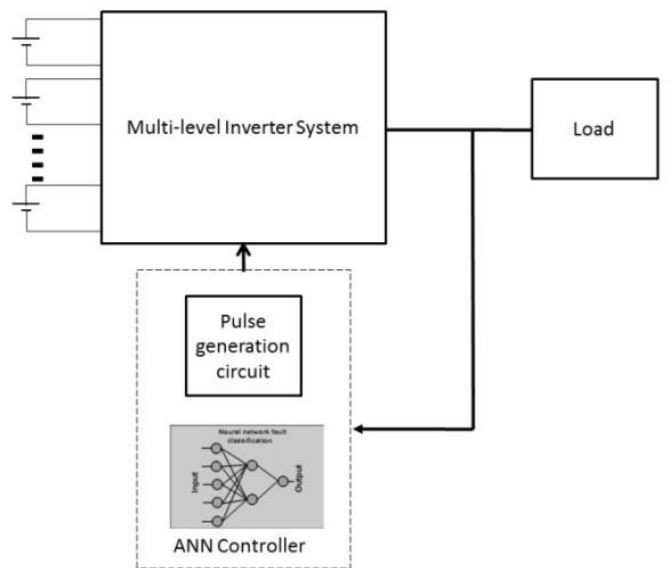

Figure 4. General Block diagram of an ANN controller based fault diagnostic system.

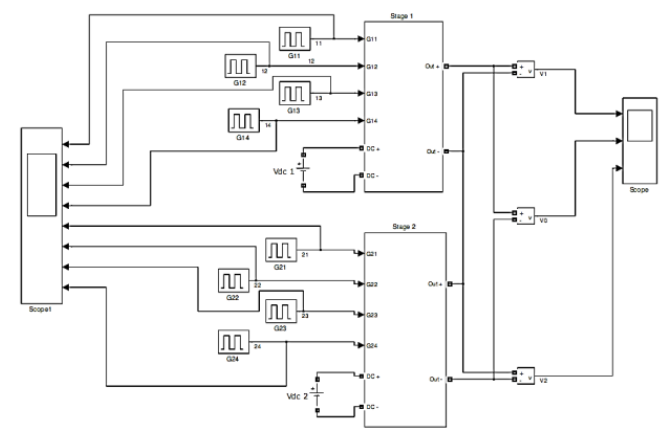

(a)

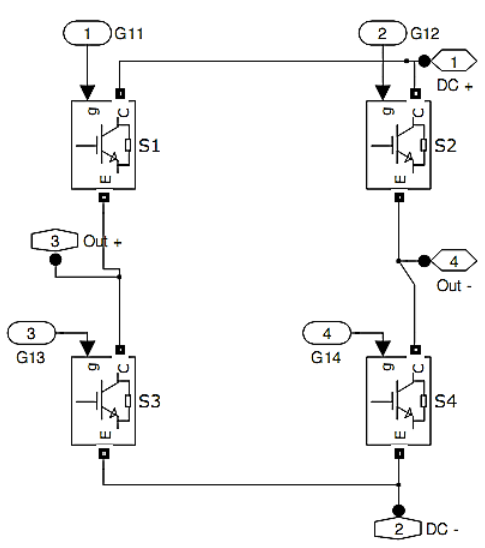

(b)

Figure 5. (a) Single stage of an MLI (b) Simulink model of a 5-level Cascaded MLI

The 5-level cascaded multi-level inverter is simulated for various irregularities in MLI outputs due to misfiring of the switches 
using Simulink as shown in figure 5. The pulses are generated in such a manner that,when a switch is misfired or triggered before it is actually meant to be triggered, there exists a conduction overlap with the second switch from the same leg which leads to appreciable distortion in the output waveform of the MLI. This distortion is noticed to be unique to each of type of the faults due to misfiring.

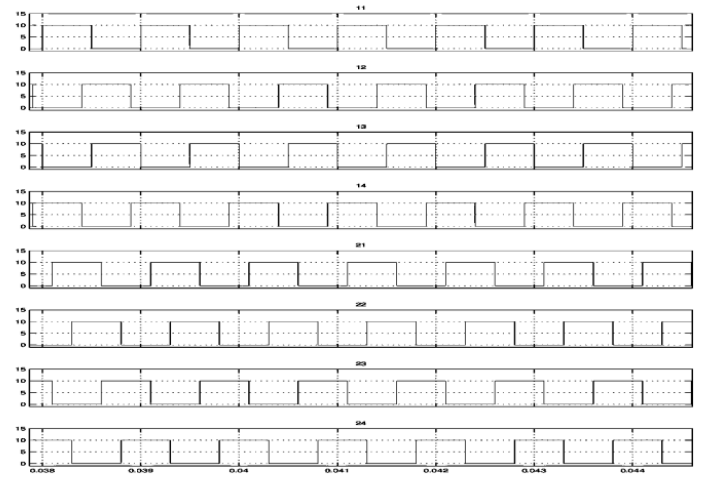

Figure 6. Firing pulses to the healthy MLI

The firing pulses and the simulated output waveforms of the healthy MLI are shown in figure 6 and figure 7 respectively. The upper and the lower waveforms in Figure 7 depict the output of the individual stages and the middle waveform gives the total output of the MLI.

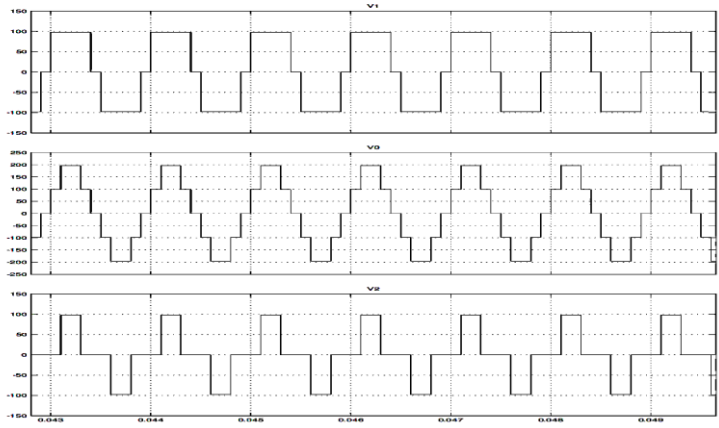

Figure 7. Output waveforms of a healthy 5-level Cascaded MLI.

\subsection{Simulations of Training Patterns}

The output waveform patterns of the MLI under faulty conditions are obtained by generating the firing pulses to the MLI in such a manner that combinations of switches are fired prior to their actual instant. To obtain the input data to train the neural network, the following combinations of misfiring are simulated:

Switch $S_{13}$ is triggered such that there is a conduction overlap between switches $S_{11}$ and $S_{13}$.

x Misfiring causing conduction overlap in switches $S_{12}$ and $\mathrm{S}_{14}$.

4 Misfiring causing conduction overlap in switches $S_{21}$ and $\mathrm{S}_{23}$.

× Misfiring causing conduction overlap in switches $S_{22}$ and $\mathrm{S}_{24}$.

A All combinations of conduction overlap in more than one pair switches, etc.
Figures 8 and 9 show few of the output patterns obtained to train the neural network.

A total number of sixteen patterns of seven thousand sample points each are obtained from all the combinations of faults due to misfiring of the switches which are given as the input training patterns to the RBF network.

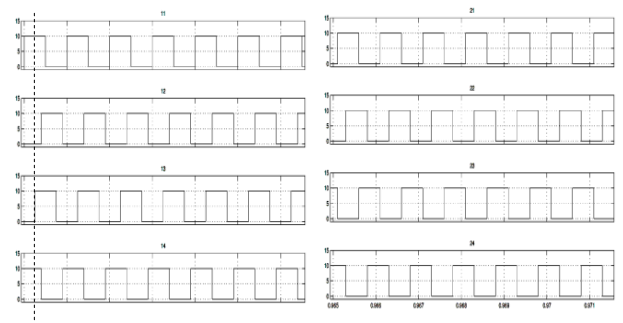

(a)

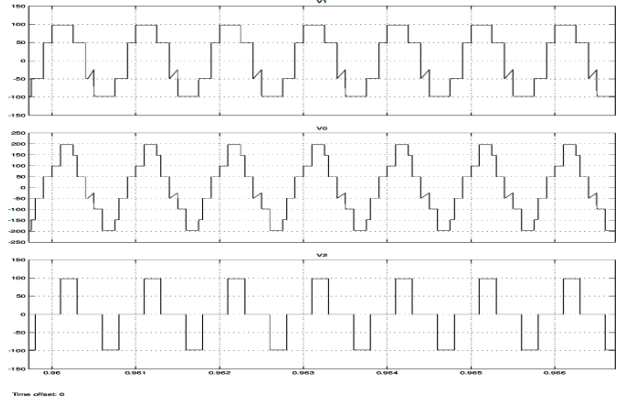

(b)

Figure 8. Triggering Pulses and Output waveforms when the Switch $S_{13}$ is triggered $0.25 \mathrm{~ms}$ early
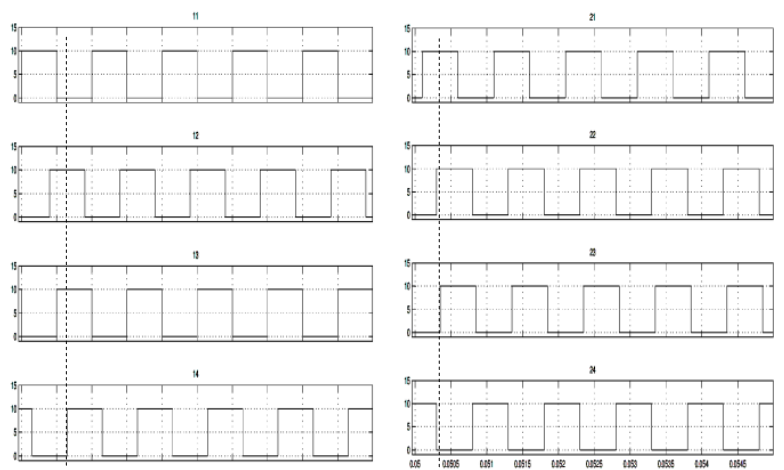

(a)

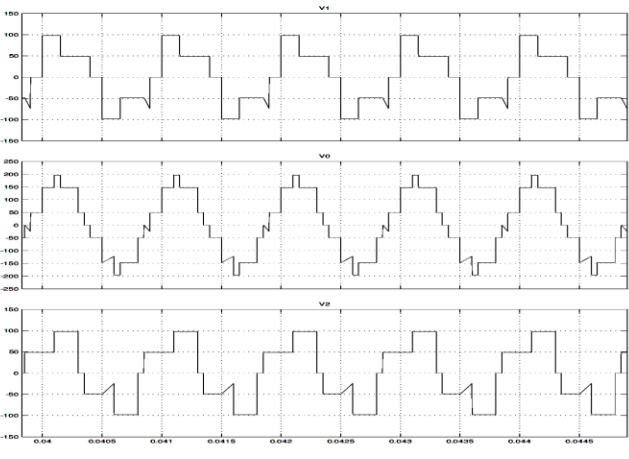

(b)

Figure 9. Firing pulses and output waveforms when switches $S_{14}$ and $S_{23}$ are fired $0.25 \mathrm{~ms}$ early 


\subsection{Training the RBF Network for Fault diagnosis}

The training patterns thus obtained by simulating the faults are fedto the RBF network generated using newrbe. The generated RBF net contains 7000 input neurons with the Gaussian function as the RBF activation function with a spread parameter of 1 and 12 output neurons with a purelin activation function. The output layer contains 12 neurons as there 12 unique types of faults that can be caused due to misfiring of the various combinations of switches in the MLI.The training target values are as shown in table 1. Each column represents each type of fault taken in the order of the input patterns provided for training.

The trained neural network is tested in two categories. First, the test vectors are selected from the training vectors and the output of the neural networks is observed. As shown in table 1, the ANN is able to identify the type of the fault and location of the fault occurring on the MLI.To test the trained neural network further, new pattern of data are simulated and provided to the input layer of the net. The pattern of the new data is similar but not identical any of the training data. The net was able to match the new test pattern to the correct pattern.

\subsection{Results Discussion}

To train the net, patterns corresponding to faults with conduction overlap period of $45^{\circ}$ are applied to the input layer of the generated RBFN. As stated, the net is tested by applying test input patterns obtained by simulating a fault of any combination mentioned in table 1 . Figure 10 gives the output of the of the neural network when the patterns corresponding to misfiring fault on switch combinations $\mathrm{S}_{12} \mathrm{~S}_{14}, \mathrm{~S}_{11} \mathrm{~S}_{13} \mathrm{~S}_{21} \mathrm{~S}_{23}$ and all pair of switches with conduction overlap of $45^{0}$ are applied which are used to train the net.

Table 1. Targets Patterns for various faults

\begin{tabular}{|c|c|}
\hline FAULT & TARget Pattern \\
\hline No fault & {$\left[\begin{array}{llllllllllll}1 & 0 & 0 & 0 & 0 & 0 & 0 & 0 & 0 & 0 & 0 & 0\end{array}\right]$} \\
\hline $\mathrm{S}_{11} \mathrm{~S}_{13}$ & 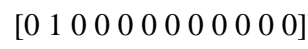 \\
\hline $\mathrm{S}_{12} \mathrm{~S}_{14}$ & {$\left[\begin{array}{llllllllllll}0 & 0 & 1 & 0 & 0 & 0 & 0 & 0 & 0 & 0 & 0 & 0\end{array}\right]$} \\
\hline $\mathrm{S}_{21} \mathrm{~S}_{23}$ & {$\left[\begin{array}{llllllllllll}0 & 0 & 0 & 1 & 0 & 0 & 0 & 0 & 0 & 0 & 0 & 0\end{array}\right]$} \\
\hline $\mathrm{S}_{22} \mathrm{~S}_{24}$ & {$\left[\begin{array}{llllllllllll}0 & 0 & 0 & 0 & 1 & 0 & 0 & 0 & 0 & 0 & 0 & 0\end{array}\right]$} \\
\hline$S_{11} S_{13} S_{21} S_{23}$ & {$\left[\begin{array}{llllllllllll}0 & 0 & 0 & 0 & 0 & 1 & 0 & 0 & 0 & 0 & 0 & 0\end{array}\right]$} \\
\hline $\mathrm{S}_{12} \mathrm{~S}_{14} \mathrm{~S}_{22} \mathrm{~S}_{24}$ & 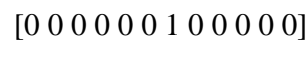 \\
\hline $\mathrm{S}_{11} \mathrm{~S}_{13} \mathrm{~S}_{22} \mathrm{~S}_{24}$ & {$\left[\begin{array}{llllllllllll}0 & 0 & 0 & 0 & 0 & 0 & 0 & 1 & 0 & 0 & 0 & 0\end{array}\right]$} \\
\hline$S_{12} S_{14} S_{21} S_{23}$ & {$\left[\begin{array}{llllllllllll}0 & 0 & 0 & 0 & 0 & 0 & 0 & 0 & 1 & 0 & 0 & 0\end{array}\right]$} \\
\hline $\mathrm{S}_{11} \mathrm{~S}_{13} \mathrm{~S}_{12} \mathrm{~S}_{14}$ & {$\left[\begin{array}{llllllllllll}0 & 0 & 0 & 0 & 0 & 0 & 0 & 0 & 0 & 1 & 0 & 0\end{array}\right]$} \\
\hline$S_{21} S_{23} S_{22} S_{24}$ & {$\left[\begin{array}{llllllllllll}0 & 0 & 0 & 0 & 0 & 0 & 0 & 0 & 0 & 0 & 1 & 0\end{array}\right]$} \\
\hline All pairs & 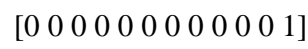 \\
\hline
\end{tabular}

Figure 11 gives the output of the net when an output voltage waveform pattern of the MLI corresponding to a misfire fault with conduction overlap period of $72^{0}$ between on pair $S_{11} S_{13}$. Though this pattern is an unknown pattern to the net, it is able to correctly associate with the correct type of misfiring fault based on the training patterns.

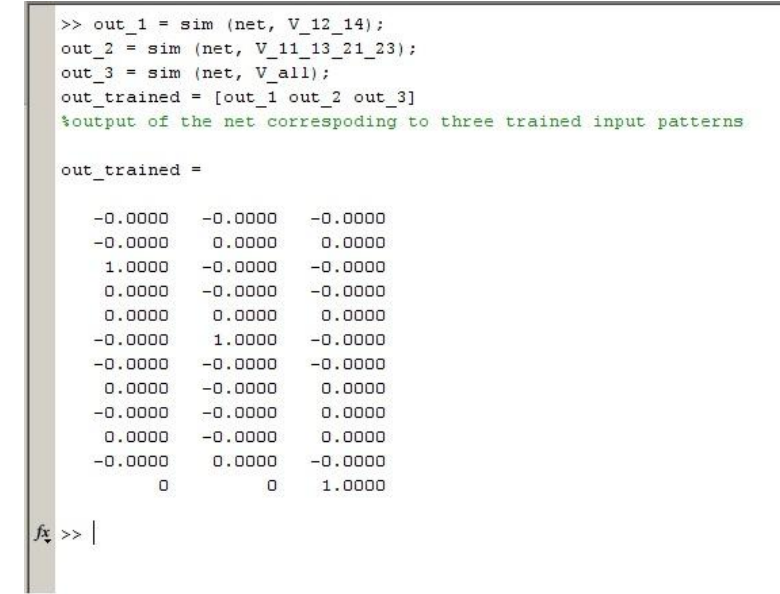

Figure 10. Output of the net corresponding to trained patterns

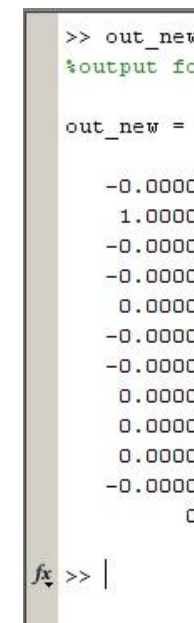

Figure 11. Output of the net to the new pattern corresponding to $72^{0}$ conduction overlap misfire fault on switch pair $S_{11} S_{13}$

\section{CONCLUSION AND FUTURE SCOPE}

A fault diagnostic system to identify faults due to misfiring of switches in a 5-level Cascaded H-Bridge Multi-level Inverter using Artificial Neural Network is proposed. A Radial Basis Function Neural Network is trained with the patterns of the output voltage waveforms during various instances of misfiring of one or more switches in the MLI. Once the network is trained with few training patterns, it is ready to identify the location(s) of the misfiring irrespective of the duration and instance of the misfiring of the switches. The neural network delivered an appreciable performance throughout the operating range of the MLI. It was also observed that, a radial basis function neural network trained with the output waveform pattern is able to efficiently identify the location(s) of the switches that were misfired when compared to training a perceptron model with either the harmonic spectrum [7] or the voltage histogram [8] or any other method.These methods were able to identify the fault only as either an open circuit fault or a short circuit fault but failed to further classify these faults as stated in Section 1. Thus, an ANN trained with wave shape of the output pattern provides a superior control over the firing pulses enabling the triggering circuitry to automatically adjust the duty cycle/ pulse width or any required parameter such that the MLI continues to produce a healthy output despite switches being misfired accidentally. As the response of an RBFN is quicker than most of the available ANN topologies, the distortion of the output during this response 
time does not have a considerable impact on the performance of the load systems and hence can be neglected.

The proposed RBF Neural Network can be trained further to identify and provide compensation for any type of faults on the MLI stated in Section 1. The future scope of the presented work includes implementing the proposed RBFN to diagnose all switch level faults, shorting and disconnecting of DC sources and load end faults. The future scope also includes implementing a Feed Forward Neural Network and a Cascade Forward Neural Network trained using the Back Propagation Training Algorithms to diagnose the faults implemented for an RBFN and present comparative study of performance of the said Neural Network topologies for the faults under consideration.

\section{REFERENCES}

[1] Surin Komfoi and Leon M. Tolbert, "Multilevel Power Converters", Chapter 31, Power Electronics Handbook, M. H. Rashid, Academic Press, 2006.

[2] Surin Komfoi, Chatrchai Aimsaard, "A 5-Level Cascaded Hybrid Multi-level Inverter for Interfacing with Renewable Energy Resources", ECTI-CON 2009, pp. 284-287

[3] Mariusz Malinowski, K. Gopakumar, Jose Rodriguez and Marcelo A. Perez, "A Survey on Cascaded Multilevel Inverters", IEEE Trans. on Industrial Electronics, Vol. 57, no. 7, pp 2197-2208, July 2010.

[4] Ebrahim Babaei and Seyed Hossein Hosseini, "New Cascaded Multilevel Inveter topology with minimum number of switches", Journal on Energy Conversion and Management, vol. 50 pp 2761-2767, July 2009, Elsevier.

[5] Pablo Lezana et al, "Survey on Fault Operation on Multilevel Inverters", IEEE Trans. on Industrial Electronics, Vol. 57, no. 7, pp 2207-2218, July 2010
[6] Laurene Fausett, "Fundamentals of Neural Networks: Architectures, Algorithms and Applications", PrenticeHall, 1994

[7] Surin Komfoi and Leon M. Tolbert, "Fault Diagnostic System for a Multilevel Inverter using a Neural Network", IEEE Trans. on Power Electronics, Vol. 22, no. 3, pp 1062-1069, May 2007

[8] S. Sedghi, A. Dastfan, A. Ahmedyfard, "Fault Diagnosis Seven Level Modular Multilevel Inverter via Voltage Histogram and Neural Network", IEEE Proc. on ICPE \& ECCE 2011, pp1005-1012, May-June, 2011.

[9] Min-Huei Kim, M. Godoy Simoes and Bimal K. Bose, "Neural Network Based Estimation of Power Electronic Waveforms", IEEE Trans on Power Electronics, Vol. 11, no. 2, pp 383-389, March 1996.

[10] R. Taleb and A. Meroufel, "Control of Asymmetrical Multilevel Inverter using Artificial Neural Network", Elektronika Ir Elektrotechnika, Vol. 96, no. 8, 2009.

[11] Backpropagation,http://en.wikipedia.org/wiki/Backpropa gation, $13^{\text {th }}$ September, 2011.

[12] Perceptron, http://en.wikipedia.org/wiki/Perceptron, $15^{\text {th }}$ September, 2011.

[13] RBF Neural Networks, http://www.dtreg.com/rbf.htm, $18^{\text {th }}$ October, 2011

[14] Simon Cohen and Nathan Intrator, "A Hybrid projection based and radial basis function", IWMCS 2000, June 2000.

[15] Radial Basis Function Network, http://en.wikipedia.org/wiki/Radial_basis_function_netw ork, $18^{\text {th }}$ October, 2011. 